\title{
Emisiones generadas por el consumo de leña y carbón en la preparación de comida rápida
}

\section{Emissions generated by firewood and charcoal consumption in fast-food preparation}

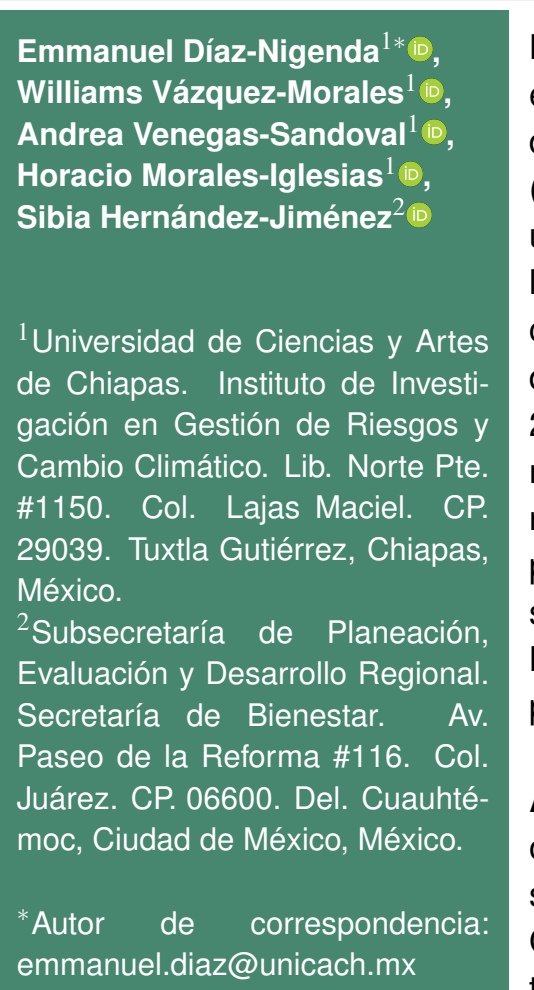

emmanuel.diaz@unicach.mx

Nota científica

Recibida: 04 de marzo 2021

Aceptada: 25 de mayo 2021

Como citar: Díaz-Nigenda E, Vázquez-Morales W, VenegasSandoval A, Morales-Iglesias $\mathrm{H}$, Hernández-Jiménez S (2021) Emisiones generadas por el consumo de leña y carbón en la preparación de comida rápida. Ecosistemas y Recursos Agropecuarios 8(2): e2962. DOI: 10.19136/era.a8n2.2962
RESUMEN. El objetivo del trabajo fue cuantificar las emisiones de gases de efecto invernadero (GEI) y otros contaminantes generados por el consumo de leña y carbón en la preparación de pollos asados en Tuxtla Gutiérrez (TGZ), Chiapas, mediante la aplicación de factores de emisión (FE). Se hizo una categorización de los establecimientos en función a su producción y la infraestructura utilizada para la actividad para determinar el combustible consumido anualmente. Los gases con emisiones más altas fueron el dióxido de carbono $\left(\mathrm{CO}_{2}, 5312.12 \mathrm{t}\right.$ año $\left.{ }^{-1}\right)$ y el monóxido de carbono (CO, $290.13 \mathrm{t}$ año $^{-1}$ ); mientras que las partículas con diámetros aerodinámicos menores a $10 \mu \mathrm{m}\left(\mathrm{PM}_{10}\right)$ fueron las más abundantes $\left(27.23 \mathrm{t} \mathrm{año}^{-1}\right)$. Los resultados muestran la relevancia del consumo de leña y carbón en la preparación de pollos asados como fuente de contaminación del aire que se suma a la problemática ambiental de la ciudad.

Palabras clave: Alimentos, biocombustible sólido, factor de emisión, gei, pollos asados.

ABSTRACT. The objective of this work was to quantify the emissions of greenhouse gases (GHG) and other pollutants generated by the consumption of firewood and charcoal in the roast chicken preparation in Tuxtla Gutiérrez (TGZ), Chiapas, by applying emission factors (EF). A categorization of establishments was made based on their production and infrastructure used for the activity and then the fuel consumed annually was determined. The gases with higher emissions were carbon dioxide $\left(\mathrm{CO}_{2}, 5312.12 \mathrm{t} \mathrm{yr}^{-1}\right)$ and carbon monoxide $\left(\mathrm{CO}, 290.13 \mathrm{t} \mathrm{yr}^{-1}\right)$; while particles with aerodynamic diameters less than $10 \mu \mathrm{m}\left(\mathrm{PM}_{10}\right)$ were the most abundant $\left(27.23 \mathrm{t} \mathrm{yr}^{-1}\right)$. Results show the relevance of firewood and charcoal consumption in roast chicken preparation as an air pollution source that adds up to environmental problems of the city.

Key words: Food, solid biofuel, emission factor, ghg, roast chicken. 


\section{INTRODUCCIÓN}

La contaminación del aire es uno de los problemas que afecta a diferentes ciudades en el mundo, cuya complejidad está definida por los impactos en la salud de la población como resultado de la exposición a altas concentraciones de contaminantes (Páramo 2019), así como de las implicaciones climáticas generadas por las emisiones de GEI, mismas que generan cambios en el clima global (Ramanathan y Feng 2009). Los contaminantes atmosféricos pueden ser emitidos por una gran diversidad de fuentes que afectan la calidad del aire de una región (SEMARNAT 2013). En la industria alimentaria, se puede hacer uso de gas LP y/o de biomasa (leña y carbón) (Masera et al. 2011) en la preparación de alimentos. El sistema alimentario a través de su cadena de suministro (producción, transporte, comercialización y preparación) contribuye significativamente a las emisiones globales de GEI (Garnett 2011).

Algunos estudios sobre el uso de leña y carbón en la cocción de alimentos cuantifican las emisiones generadas en función del alimento (contenido de grasas) y condimentos utilizados para su preparación (McDonald et al. 2003). Otros evalúan la eficiencia energética de estufas domésticas y su relación con las emisiones de GEI y otros contaminantes (Smith 1994, Bhattacharya y Abdul-Salam 2002). Además, se ha buscado determinar los factores de emisión para gases y partículas como resultado del uso de diferentes tipos de biomasa como combustible (Lango-Reynoso et al. 2018, Andreae 2019, PadillaBarrera et al. 2019).

Debido a la dinámica de la sociedad, con jornadas laborales cada vez más exigentes, demandantes y absorbentes, los alimentos preparados son una forma de satisfacer las necesidades alimentarias de la población, por lo que la comida rápida es una de las principales opciones de alimentación de las personas (Vilaplana 2002). La producción de alimentos preparados es comúnmente practicada en México, en donde se emplean usualmente asadores de uso comercial que utilizan como combustible leña o carbón (Lango-Reynoso et al. 2018, Padilla-Barrera et al. 2019). En consecuencia, los contaminantes generados pueden ser diversos, sin embargo, es común encontrar $\mathrm{CO}_{2}, \mathrm{CO}$, metano $\left(\mathrm{CH}_{4}\right)$, óxido nitroso $\left(\mathrm{N}_{2} \mathrm{O}\right)$, óxidos de nitrógeno $\left(\mathrm{NO}_{x}\right)$, compuestos orgánicos totales diferentes al metano (TNMOC por sus siglas en inglés), hidrocarburos totales diferentes al metano (TNMHC por sus siglas en inglés), partículas suspendidas totales (PST), $\mathrm{PM}_{10}$ y $\mathrm{PM}_{2.5}$, entre otros que afectan la calidad del aire y la salud de la población (IEECC 2015). En Tuxtla Gutiérrez, Chiapas, la producción de alimentos preparados, referidos a pollos asados, ha tenido un aumento considerable debido al crecimiento acelerado de la ciudad, lo cual demanda un mayor consumo de combustibles y, por consiguiente, un incremento en las emisiones de contaminantes a la atmósfera. Con base a lo anterior el objetivo planteado fue cuantificar las emisiones de GEI y otros contaminantes generados por el consumo de leña y carbón en la preparación de pollos asados en TGZ.

\section{MATERIALES Y MÉTODOS}

\section{Descripción del área de estudio}

La ciudad de TGZ se ubica dentro de las coordenadas: $16^{\circ} 40^{\prime} 30^{\prime \prime}$ y $16^{\circ} 48^{\prime} 00^{\prime \prime} \mathrm{LN}, 93^{\circ} 01^{\prime}$ $35^{\prime \prime}$ y $93^{\circ} 13^{\prime} 10^{\prime \prime}$ LO, a una altitud de 522 metros sobre el nivel del mar, en la parte centro-occidente del estado de Chiapas, México (Figura 1). Ocupa una superficie de $334.61 \mathrm{~km}^{2}$ en la cuenca baja del río Sabinal, al norte colinda con las montañas del Parque Nacional Cañón del Sumidero, al este con el río Grijalva y Chiapa de Corzo, al sur con la Mesa de Copoya y el Cerro Mactumatzá, al oeste con las montañas que integran la cuenca alta (CEIEG 2020). Con una población de 604147 habitantes al 2020 (INEGI 2020), TGZ ha tenido un crecimiento acelerado y desordenado en los últimos años, lo que favorece la presencia de calles sin pavimentar las cuales emiten partículas que afectan la calidad del aire de la localidad (Díaz-Nigenda et al. 2018). La dinámica de las actividades propias de una ciudad, hacen que la producción y preparación de alimentos que emplean leña o carbón tengan un crecimiento considerable, que se suma al transporte, los incendios forestales y 


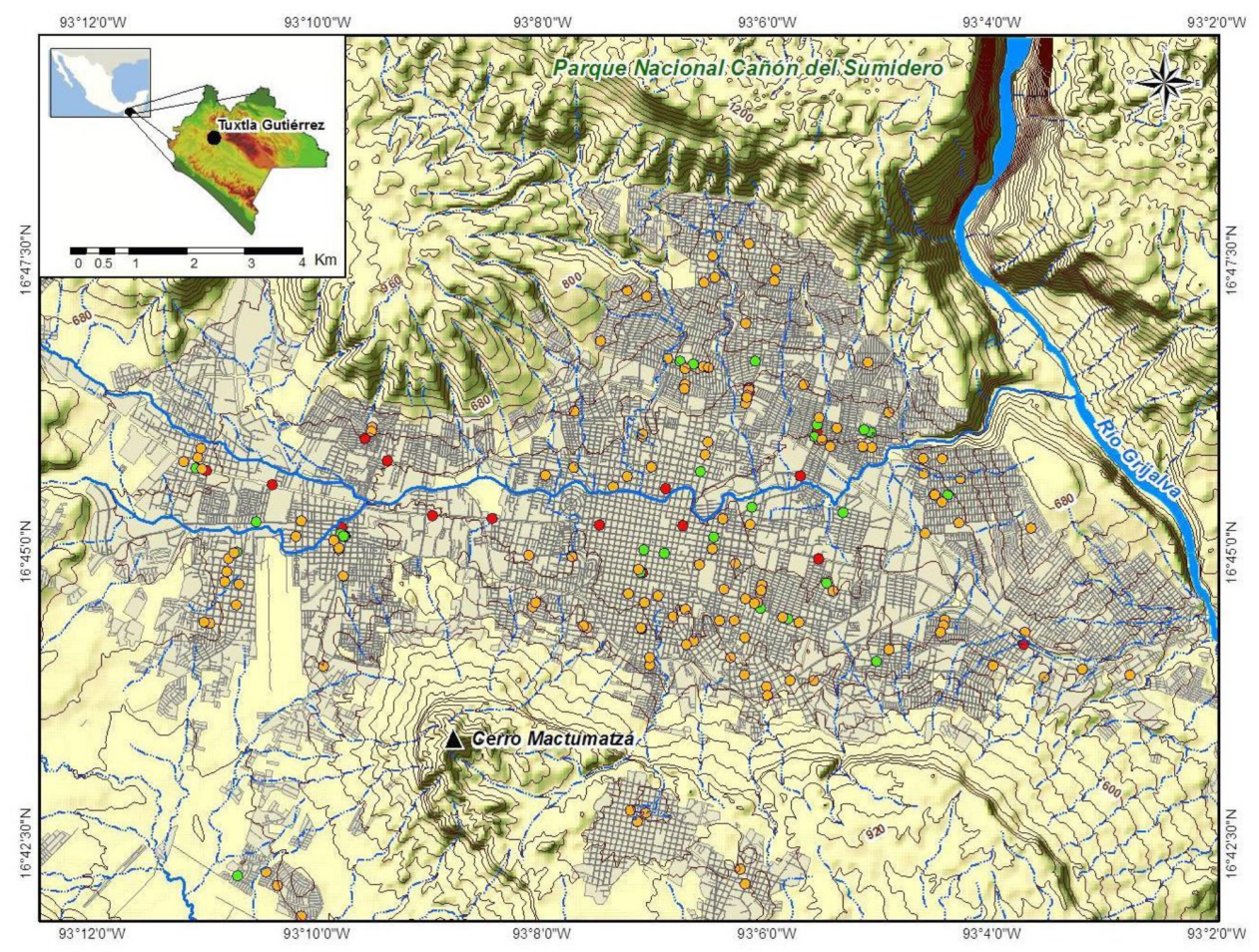

Figura 1. Zona de estudio y ubicación de establecimientos en la ciudad de Tuxtla Gutiérrez. Establecimientos pequeños (amarillo), medianos (verde) y grandes (rojo).

quemas de predios, como fuentes de contaminación.

\section{Datos de actividad}

El número de establecimientos que se dedicaban a la producción y preparación de alimentos (asado de pollos) fue obtenido del Directorio Estadístico de Unidades Económicas (DENUE) del Instituto Nacional de Estadística y Geografía (INEGI 2014). La información fue validada a través de visitas durante el año 2014. Se encontraron 166 establecimientos en operación, además de 11 locales que no aparecían en los registros, por lo que un total de 177 comercios fueron considerados en el estudio.

\section{Aplicación de encuestas}

Se aplicaron encuestas con preguntas relacionadas a datos del establecimiento, tipo de combustible utilizado, combustible consumido $(\mathrm{kg})$, producción diaria, días y horario de servicio. Los establecimientos se clasificaron en tres categorías con base a su producción e infraestructura (Tabla 1); identificándose franquicias nacionales, así como locales establecidos a orillas de calles y avenidas. De acuerdo con Aguilar-Barojas (2005), el número de establecimientos encuestados fue calculado por:

$$
n_{i}=\frac{N_{i} \sigma^{2} Z^{2}}{\left(N_{i}-1\right) e^{2}+\sigma^{2} Z^{2}}
$$

Donde: $\mathrm{i}$ = categoría, $\mathrm{n}_{i}=$ tamaño de la muestra en la categoría $\mathrm{i}, \mathrm{N}_{i}=$ número de establecimientos en la categoría $\mathrm{i}, \mathrm{Z}=$ valor de 1.96 para un nivel de confianza del $95 \%, \sigma=$ varianza de la población en estudio $(0.5)$ y e $=$ nivel de precisión absoluta $(9 \%)$.

\section{Estimación de combustible consumido}

El consumo anual por categoría fue calculado con la expresión:

$$
C A_{i}=C P_{i} \times N_{i} \times 365
$$

Donde: $\mathrm{i}$ = categoría, $\mathrm{CA}_{i}=$ consumo anual de combustible en la categoría i $\left(\mathrm{kg}\right.$ año $\left.{ }^{-1}\right), \mathrm{CP}_{i}=$ consumo 
Tabla 1. Información de establecimientos dedicados al asado de pollos.

\begin{tabular}{lccccc}
\hline Categoría & Combustible & $\begin{array}{c}\text { Producción } \\
\left(\text { pollos día }^{-1}\right)\end{array}$ & $\begin{array}{c}\text { Número de } \\
\text { establecimientos }\end{array}$ & $\begin{array}{c}\text { Consumo diario por } \\
\text { establecimiento }\left(\mathrm{kg} \mathrm{d}^{-1}\right)\end{array}$ & $\begin{array}{c}\text { Consumo anual por } \\
\text { categoría }\left(\mathrm{kg}_{\text {año }}{ }^{-1}\right)\end{array}$ \\
\hline Pequeño & Carbón & $<50$ & 132 & $15.94( \pm 7.95)$ & 767989.20 \\
Mediano & Leña & $50-100$ & 28 & $138.91( \pm 52.20)$ & 1419660.20 \\
Grande & Carbón & $>100$ & 17 & $81.25( \pm 28.37)$ & 504156.25 \\
TOTAL & - & - & 177 & - & - \\
\hline Datos entre paréntesis indican la desviación estándar. & & &
\end{tabular}

promedio por establecimiento en la categoría i ( $\mathrm{kg}$ $\mathrm{d}^{-1}$ ) y $\mathrm{N}_{i}=$ número de establecimientos en la categoría i.

\section{Cuantificación de emisiones}

El uso de FE representa una técnica rápida para el cálculo de emisiones de contaminantes, por lo que se cuantificaron las descargas anuales de $\mathrm{CO}_{2}$, $\mathrm{CH}_{4}, \mathrm{~N}_{2} \mathrm{O}, \mathrm{CO}, \mathrm{NO}_{x}$, TNMOC, TNMHC, PST, $\mathrm{PM}_{10} \mathrm{y}$ $\mathrm{PM}_{2.5}$. Lo anterior fue realizado mediante la ecuación (SEMARNAT 2013):

$$
E_{i j}=C A_{i} \times F E_{j} \times \frac{1 \text { ton }}{10^{6} g}
$$

Donde: $\mathrm{i}$ = categoría, $\mathrm{j}$ = contaminante, $\mathrm{E}_{i j}=$ emisión anual del contaminante $\mathrm{j}$ en la categoría $\mathrm{i}\left(\mathrm{t}\right.$ año ${ }^{-1}$ ), $\mathrm{CA}_{i}=$ consumo anual de combustible en la categoría i $\left(\mathrm{kg}^{\mathrm{año}}{ }^{-1}\right)$ y $\mathrm{FE}_{j}=$ factor de emisión del contaminante j por tipo de combustible $\left(\mathrm{g} \mathrm{kg}^{-1}\right)$. Los FE empleados por tipo de combustible se tomaron de Smith (1994), EPA (1995), IPCC (1997), Zhang et al. (2000), Andreae y Merlet (2001), Battacharya y Abdul-Salam (2002), Bertschi et al. (2003), Yokelson et al. (2008), Christian et al. (2010), Akagi et al. (2011), Padilla-Barrera et al. (2019) y Andreae (2019), y se muestran en las Tablas 2 y 3 . De ellos se obtuvo un FE promedio $\left(\mathrm{FE}_{p}\right)$ para cada contaminante, así como su desviación estándar.

\section{RESULTADOS Y DISCUSIÓN}

Se consideraron 177 comercios en el estudio, formando tres categorías con base a la infraestructura con la que contaban los locales al momento de la encuesta: los grandes fueron franquicias de empresas nacionales con asadores eficientes en el uso del combustible; los medianos consideraban locales con asadores semi estructurados en ladrillos; mientras que los pequeños fueron aquellos negocios con asadores metálicos de menor tamaño. En la Figura 1 se observa que los locales pequeños y medianos, no tienen un patrón de distribución definido, por el contrario, los grandes se localizan a lo largo de una franja central que atraviesa la ciudad.

Se aplicaron 101 encuestas distribuidas de la siguiente manera: 15 en establecimientos grandes, 23 en comercios medianos y 63 en establecimientos pequeños. Con la información obtenida se estimó la cantidad promedio de combustible consumido anualmente por cada una de las categorías. Los establecimientos pequeños $\left(R^{2}=0.68\right)$ y grandes $\left(R^{2}\right.$ $=0.63$ ), tienen una relación directa con el consumo de carbón y la producción de pollos asados. Mientras que en los establecimientos medianos, no se identificó este comportamiento con el uso de la leña, lo cual se atribuye a la falta de control en el uso de este combustible, ya que utilizan cualquier tipo de vegetación sin importar su poder calorífico. Lo anterior, sumado al diseño irregular de los asaderos, generan que el consumo de leña de los establecimientos medianos presente mayor variación $\left( \pm 52.2 \mathrm{~kg} \mathrm{~d}^{-1}\right)$ en comparación con las otras categorías. Los establecimientos medianos consumen aproximadamente $1419 \mathrm{t}$ año $^{-1}$ de leña, mientras que los pequeños queman cerca de $768 \mathrm{t}^{\mathrm{año}}{ }^{-1}$ y los grandes $504 \mathrm{t}$ año ${ }^{-1}$ de carbón (Tabla 1).

En las Tablas 2 y 3 , se observa que los $\mathrm{FE}_{p}$ para $\mathrm{CO}_{2}, \mathrm{CO}, \mathrm{N}_{2} \mathrm{O}$ y $\mathrm{NO}_{x}$ del carbón, son mayores a los de la leña, sin embargo, las desviaciones son más grandes, lo que coincide con Andreae y Merlet (2001). Pero de acuerdo con Zhang et al. (2000) y Akagi et al. (2011), se observó lo contrario para TNMOC, TNMHC, PST y $\mathrm{PM}_{10}$, donde los $\mathrm{FE}_{p}$ son mayores para la leña. Para $\mathrm{CH}_{4}$ y $\mathrm{PM}_{2.5}$, como lo 
Tabla 2. Factores de emisión de contaminantes empleados para el cálculo de emisiones por el consumo de carbón.

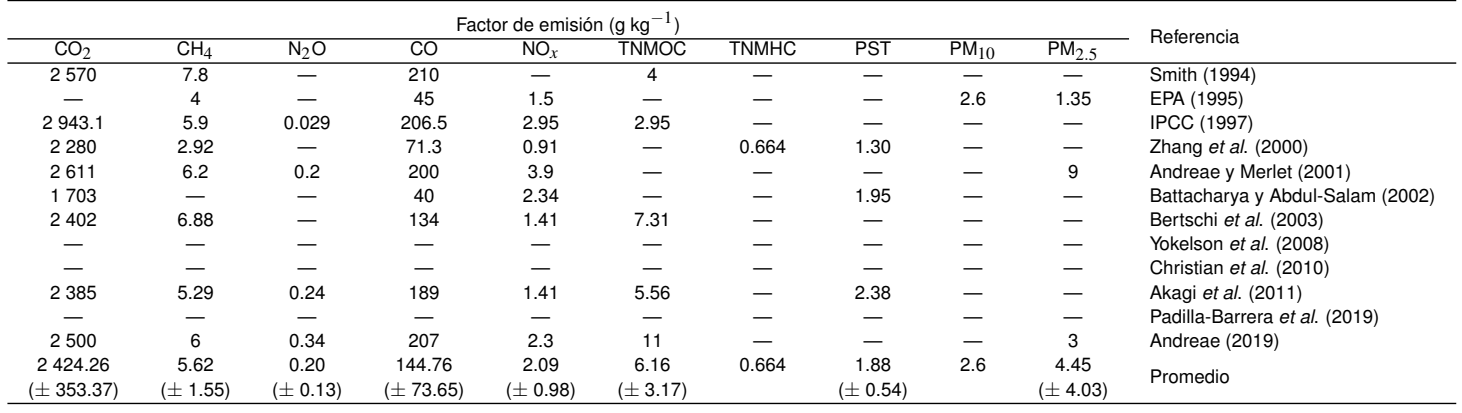

Tabla 3. Factores de emisión de contaminantes empleados para el cálculo de emisiones por el consumo de leña.

\begin{tabular}{|c|c|c|c|c|c|c|c|c|c|c|}
\hline \multicolumn{10}{|c|}{ Factor de emisión $\left(\mathrm{g} \mathrm{kg}^{-1}\right)$} & \multirow{2}{*}{ Referencia } \\
\hline $\mathrm{CO}_{2}$ & $\mathrm{CH}_{4}$ & $\mathrm{~N}_{2} \mathrm{O}$ & $\mathrm{CO}$ & $\mathrm{NO}_{x}$ & TNMOC & TNMHC & PST & $\mathrm{PM}_{10}$ & $\mathrm{PM}_{2.5}$ & \\
\hline 1700 & 4.8 & - & 115.4 & 1.4 & 26.5 & - & - & 15.3 & 1.84 & EPA (1995) \\
\hline 1520 & 5.06 & - & 69.2 & 1.19 & - & 4.34 & 3.82 & - & - & Zhang et al. (2000) \\
\hline 1550 & 6.1 & 0.06 & 78 & 1.1 & - & - & - & - & 7.2 & Andreae y Merlet (2001) \\
\hline 1609 & 3.95 & 0.049 & 38.2 & - & - & - & - & - & - & Battacharya y Abdul-Salam (2002) \\
\hline 1525 & 10.4 & - & 96 & 2.04 & 26.78 & - & - & - & - & Bertschi et al. (2003) \\
\hline 1548 & 4.86 & - & 77 & 1.42 & 19.2 & 2.27 & 4.55 & 18.4 & 6.64 & Akagi et al. (2011) \\
\hline 1364 & 3.2 & - & 51.62 & 0.87 & - & - & - & - & 2.11 & Padilla-Barrera et al. (2019) \\
\hline 1550 & 6.8 & 0.07 & 83 & 1.3 & 58 & - & - & - & 6.8 & Andreae (2019) \\
\hline $\begin{array}{c}1569.5 \\
( \pm 104.25)\end{array}$ & $\begin{array}{c}5.47 \\
( \pm 2.25)\end{array}$ & $\begin{array}{c}0.06 \\
( \pm 0.01)\end{array}$ & $\begin{array}{c}74.65 \\
( \pm 21.84)\end{array}$ & $\begin{array}{c}1.38 \\
( \pm 0.34)\end{array}$ & $\begin{array}{c}22.23 \\
( \pm 17.94)\end{array}$ & 3.31 & 4.19 & 16.85 & $\begin{array}{c}5.89 \\
( \pm 2.91)\end{array}$ & Promedio \\
\hline
\end{tabular}

reportan la EPA (1995) y Andreae y Merlet (2001), los $\mathrm{FE}_{P}$ son similares para ambos combustibles. Las diferencias entre los FE utilizados resultan de las técnicas aplicadas y las características de los combustibles empleados para su determinación.

El comportamiento de las emisiones de los diferentes GEl y otros contaminantes para cada categoría se muestra en la Figura 2 y la Tabla 4. Los establecimientos medianos presentaron las descargas más altas de $\mathrm{CO}_{2}, \mathrm{CH}_{4}, \mathrm{NO}_{x}$, TNMOC, TNMHC, PST, $\mathrm{PM}_{10}$ y $\mathrm{PM}_{2.5}$, lo cual, de acuerdo con Battacharya y Abdul-Salam (2002), se debe a la cantidad de combustible consumido anualmente $y$, con base al tipo de combustible, los resultados coinciden con Akagi et al. (2011), IEECC (2015) y Lango-Reynoso et al. (2018) quienes señalan que las emisiones por el uso de leña son mayores a las del carbón.

Por el contrario, los establecimientos pequeños generaron las emisiones más altas de $\mathrm{CO}$ y $\mathrm{N}_{2} \mathrm{O}$, lo cual, considerando la diferencia en la cantidad de combustible consumido (Tabla 1), se atribuye a los FE que, de acuerdo con Akagi et al. (2011) y Andreae (2019) son mayores para el carbón (Tablas 2 y
3). En tanto que los grandes establecimientos o franquicias presentan las emisiones más bajas, lo cual se puede deber a que el proceso de producción tiene un aprovechamiento energético más eficiente que reduce el consumo de combustible; lo que coincide con Zhang et al. (2000) y Battacharya y Abdul-Salam (2002) quienes señalan el beneficio generado por el uso de dispositivos con mejor eficiencia energética.

En la Tabla 4 se observa que el $\mathrm{CO}_{2}(5312.12$ $\mathrm{t}$ año $\left.{ }^{-1}\right)$ y el CO $\left(290.13 \mathrm{t}\right.$ año $\left.{ }^{-1}\right)$ son los gases más abundantes. Lo que es de interés debido a que en la región las tasas de deforestación y degradación de la cobertura forestal son mayores a las de recuperación (SEMAHN 2011), lo cual se encuentra relacionado con la extracción de leña. En el caso de las partículas, las más abundantes son las $\mathrm{PM}_{10}(27.23 \mathrm{t}$ año $\left.{ }^{-1}\right)$, seguidas de las $\mathrm{PM}_{2.5}\left(14.03 \mathrm{t}\right.$ año $\left.{ }^{-1}\right)$ y de las PST (8.37 $\mathrm{t}$ año $\left.{ }^{-1}\right)$; en los tres casos, los establecimientos medianos generaron más del $50 \%$ de las emisiones de cada contaminante por el consumo de leña.

Las emisiones de TNMOC y $\mathrm{NO}_{x}$ no son tan grandes como aquellas de $\mathrm{CO}_{2}$, sin embargo, su im- 

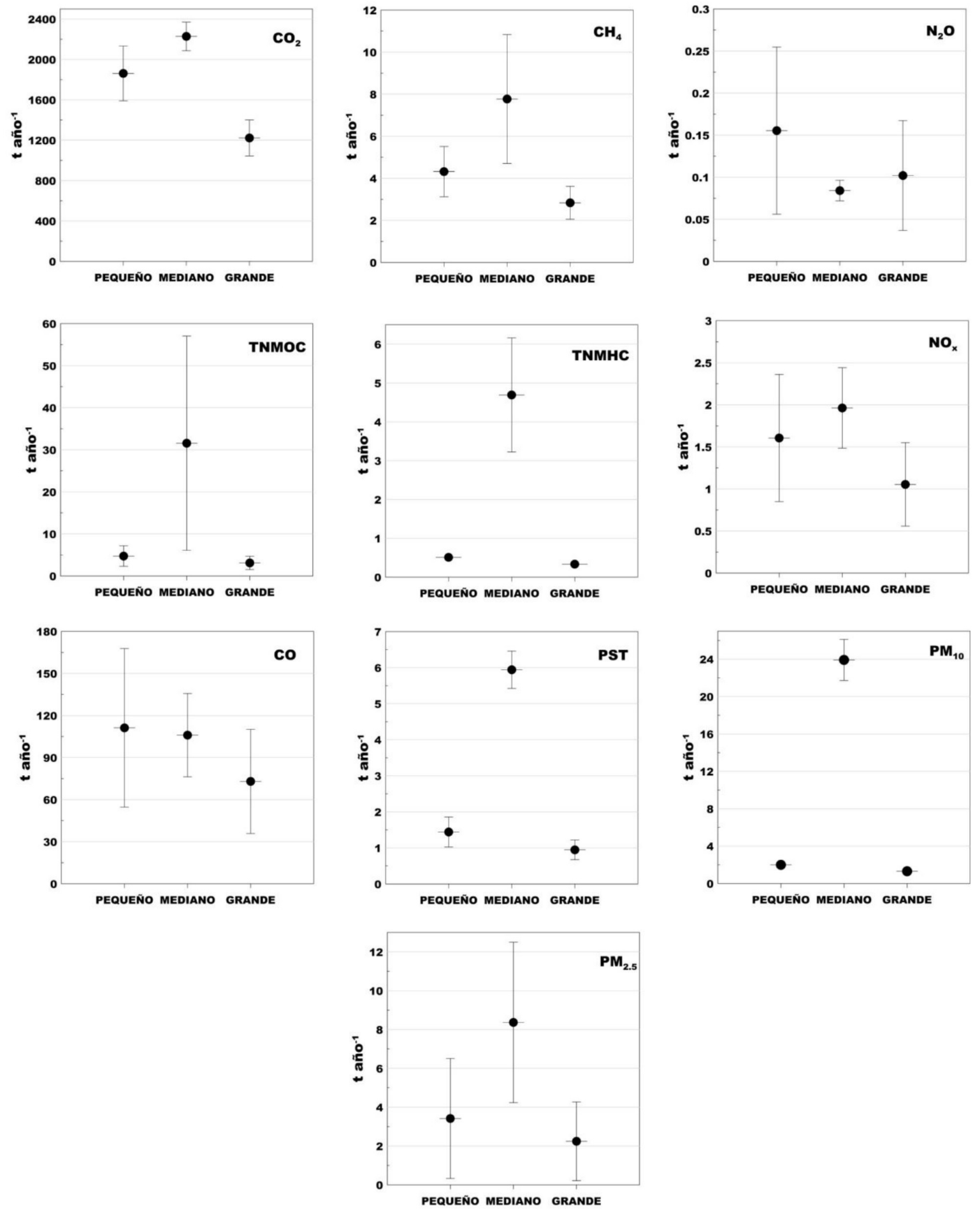

Figura 2. Comparativo de emisiones generadas por el asado de pollos en las diferentes categorías. 


\begin{tabular}{lcccccccccc}
\multicolumn{1}{l}{ Tabla 4. Emisiones anuales por tipo de contaminante $(\mathrm{t}$ año } \\
\hline Establecimientos & $\mathrm{CO}_{2}$ & $\mathrm{CH}_{4}$ & $\mathrm{~N}_{2} \mathrm{O}$ & $\mathrm{CO}$ & $\mathrm{NO}_{x}$ & $\mathrm{TNMOC}$ & $\mathrm{TNMHC}$ & $\mathrm{PST}$ & $\mathrm{PM}_{10}$ & $\mathrm{PM}_{2.5}$ \\
\hline Pequeños & 1861.81 & 4.32 & 0.16 & 111.17 & 1.61 & 4.73 & 0.51 & 1.44 & 2.00 & 3.42 \\
Medianos & 2228.11 & 7.77 & 0.08 & 105.98 & 1.96 & 31.57 & 4.69 & 5.94 & 23.92 & 8.37 \\
Grandes & 1222.21 & 2.84 & 0.10 & 72.98 & 1.05 & 3.11 & 0.33 & 0.95 & 1.31 & 2.24 \\
Total & 5312.12 & 14.93 & 0.34 & 290.13 & 4.62 & 39.41 & 5.54 & 8.33 & 27.23 & 14.03 \\
\hline
\end{tabular}

portancia radica en el rol que desempeñan como precursores para la formación de ozono (Akagi et al.2011), el cual, junto con $\mathrm{PM}_{10}$, son los principales contaminantes que afectan la calidad del aire de la ciudad.

Los resultados muestran la relevancia que tiene el consumo de leña y carbón en la preparación de pollos asados como fuente de emisión de contaminantes atmosféricos que sumado a los incen- dios forestales, quemas de predios, a las emisiones vehiculares y la resuspensión de partículas en calles sin pavimentar que se presentan en la región, permiten dimensionar y entender la problemática ambiental. Por lo que el monitoreo de la calidad del aire es necesario para desarrollar e implementar estrategias que permitan prevenir escenarios severos de contaminación atmosférica para cualquier ciudad en desarrollo.

\section{LITERATURA CITADA}

Aguilar-Barojas S (2005) Fórmulas para el cálculo de la muestra en investigaciones de salud. Salud en Tabasco 11: 333-338.

Akagi S K, Yokelson RJ, Wiedinmyer CM, Alvarado J, Reid JS, Karl T, Crounse JD, Wennberg PO (2011) Emission factors for open and domestic biomass burning for use in atmospheric models. Atmospheric Chemistry and Physics 11: 4039-4072.

Andreae MO, Merlet P (2001) Emission of trace gases and aerosols from biomass burning. Global Biogeochemical Cycles 15: 955-966.

Andreae MO (2019) Emission of trace gases and aerosols from biomass burning - an updated assessment. Atmospheric Chemistry and Physics 19: 8523-8546.

Bhattacharya SC, Abdul Salam P (2002) Low greenhouse gas biomass options for cooking in the developing countries. Biomass and Bioenergy 22: 305-317.

Bertschi IT, Yokelson RJ, Ward DE, Christian TJ, Hao WM (2003) Trace gas emissions from the production and use of domestic biofuels in Zambia measured by open-path Fourier transform infrared spectroscopy. Journal of Geophysical Research 108: 8469. DOI: 10.1029/2002JD002158

Christian TJ, Yokelson RJ, Cárdenas B, Molina LT, Engling G, Hsu SC (2010). Trace gas and particle emissions from domestic and industrial biofuel use and garbage burning in central Mexico. Atmospheric Chemistry and Physics 10: 565-584.

CEIEG (2020) Ubicación geográfica del municipio de Tuxtla Gutiérrez. Perfiles municipales. Comité Estatal de Información Estadística y Geográfica de Chiapas. Chiapas, México. http://www.ceieg.chiapas.gob.mx. Fecha de consulta: 20 de agosto de 2020.

Díaz-Nigenda E, Tatarko J, Morales-Iglesias H, Hernández MZ, Vázquez MW, Alatorre-lbargüengoitia M (2018) Measurement and Modeling Air Quality Impacts of Dust Emissions from Unpaved Roads in Tuxtla Gutiérrez, Chiapas. Geosciences 8: 284. DOI: 10.3390/geosciences8080284 
EPA (1995) Compilation of Air Pollutant Emissions Factors. AP 42, Fifth Edition. Volume 1: Stationary Point and Area Sources. Environmental Protection Agency. Research Triangle Park, NC, USA. https://www.epa.gov/airemissions-factors-and-quantification/ap-42-compilation-air-emissions-factors\#5thed. Fecha de consulta: 10 de marzo de 2020

Garnett T (2011) Where are the best opportunities for reducing greenhouse gas emissions in the food system (including the food chain)? Food Policy 36: S23-S32.

IEECC (2015) Determinación de las emisiones por la quema de combustibles en casas habitación. Instituto Estatal de Energía y Cambio Climático. Estado de México, México. 38 p. https://docplayer.es/29462748Determinacion-de-las-emisiones-por-la-quema-de-combustibles-en-casas-habitacion.html. Fecha de consulta: 15 de agosto de 2020.

INEGI (2014) Directorio Estadístico Nacional de Unidades Económicas (DENUE). Instituto Nacional de Estadística y Geografía. Chiapas, México. https://www.inegi.org.mx/app/mapa/denue/. Fecha de consulta: 20 de agosto de 2014.

INEGI (2020) Censo de Población y Vivienda 2020. Instituto Nacional de Estadística y Geografía. Chiapas, México. http://www.cuentame.inegi.org.mx/monografias/informacion/chis/poblacion/. Fecha de consulta: 13 de mayo de 2021.

IPCC (1997) Revised 1996 IPCC Guidelines for National Greenhouse Gas Inventories. Workbook Volume 2. Intergovernmental Panel on Climate Change. https://www.ipcc-nggip.iges.or.jp/public/gl/invs5.html. Fecha de consulta: 20 de marzo de 2020.

Lango-Reynoso V, López-Spiegel J, Lango-Reynoso F, Castañeda-Chávez M, Montoya-Mendoza J (2018) Estimation of $\mathrm{CO}_{2}$ emissions produced by commercial grills in Veracruz, Mexico. Sustainability 10: 464. DOI: 10.3390/su10020464

McDonald JD, Zielinska B, Fujita EM, Sagebiel JC, Chow JC, Watson JG (2003) Emissions from Charbroiling and Grilling of Chicken and Beef. Journal of the Air \& Waste Management Association 53: 185-194.

Masera CO, Coralli F, García C, Riegelhaupt E, Arias T, Vega J, Díaz R, Guerrero G, Cecotti L (2011) La bioenergía en México. Situación actual y perspectivas. Red Mexicana de Bioenergía. México. 44p.

Padilla-Barrera Z, Torres-Jardón R, Ruiz-Suarez LG, Castro T, Peralta O, Saavedra MI, Masera O, Molina LT, Zavala M (2019) Determination of emission factors for climate forcers and air pollutants from improved wood-burning cookstoves in Mexico. Energy for Sustainable Development 50: 61-68.

Páramo FVH (2019) Estado de la Calidad del Aire en México. Instituto Nacional de Ecología y Cambio Climático Blog. Ciudad de México, México. https://www.gob.mx/inecc/es/articulos/estado-de-la-calidad-del-aire-enmexico?idiom=es. Fecha de consulta: 18 de julio de 2020.

Ramanathan V, Feng Y (2009) Air pollution, greenhouse gases and climate change: Global and regional perspectives. Atmospheric Environment 43: 37-50.

SEMAHN (2011) Programa de Acción ante el Cambio Climático del estado de Chiapas. Secretaría de Medio Ambiente e Historia Natural. Chiapas, México. 137p.

SEMARNAT (2013) Guía metodológica para la estimación de emisiones de fuentes fijas. Secretaría de Medio Ambiente y Recursos Naturales. México. 146p.

Smith KR (1994) Health, energy, and greenhouse-gas impacts of biomass combustion in household stoves. Energy for Sustainable Development 1: 23-29. 
Vilaplana M (2002) Comida rápida: ¿una alternativa a la alimentación convencional? Ámbito Farmacéutico 21 : 112-118.

Yokelson RJ, Christian TJ, Karl TG, Guenther A (2008) The tropical forest and fire emissions experiment: laboratory fire measurements and synthesis of campaign data. Atmospheric Chemistry and Physics 8: 3509-3527.

Zhang J, Smith KR, Ma Y, Ye S, Jiang F, Qi W, Liu P, Khalil MAK, Rasmussen RA, Thorneloe SA (2000) Greenhouse gases and other airborne pollutants from household stoves in China: a database for emission factors. Atmospheric Environment 34: 4537-4549. 